\title{
Maximum Spreading and Rebound of a Droplet Impacting onto a Spherical Surface at low Weber numbers
}

\author{
Alireza Bordbar ${ }^{\mathrm{a}^{*}}$, Arsalan Taassob ${ }^{\mathrm{a}}$, Danial Khojasteh ${ }^{\mathrm{a}, \mathrm{b}}$, Marco Marengo ${ }^{\mathrm{c}}$, Reza Kamali ${ }^{\mathrm{a}}$ \\ ${ }^{a}$ School of Mechanical Engineering, Shiraz University, Shiraz, 71936-16548, Iran \\ ${ }^{b}$ School of Civil \& Environmental Engineering, UNSW Water Research Laboratory, 110 King St, Manly \\ Vale, NSW 2093, Australia \\ ${ }^{c}$ School of Computing, Engineering and Mathematics, University of Brighton, BN2 4GJ Brighton, United \\ Kingdom
}

\begin{abstract}
The spreading and rebound patterns of low-viscous droplets upon impacting spherical solid surfaces are investigated numerically. The studied cases consider a droplet impinging onto hydrophobic and superhydrophobic surfaces with various parameters varied throughout the study and their effects on the post-impingement behavior are discussed. These parameters include impact Weber number (through varying the surface tension and impingement velocity), the size ratio of the droplet to the solid surface, and the surface contact angle. According to the findings, the maximum spreading diameter increases with the impact velocity, with an increase of the sphere diameter, with a lower surface wettability and a lower surface tension. Typical outcomes of the impact include 1) complete rebound, 2) splash, and 3) a final deposition stage after a series of spreading and recoiling phases. Finally, a novel, practical model is proposed, which can reasonably predict the maximum deformation of low Reynolds number impact of droplets onto hydrophobic or superhydrophobic spherical solid surfaces.
\end{abstract}

${ }^{*}$ Corresponding author: alireza.bordbar@shirazu.ac.ir 
Keywords: Droplet impact, Hydrophobicity, Curved surfaces, Droplet rebound, Spreading diameter, CFD.

\section{Introduction}

Droplet impact onto solid surfaces is a phenomenon frequently observed in both nature and engineering applications. ${ }^{1}$ Common examples include impact onto plant leaves, ${ }^{2}$ internal combustion engines where fuel droplets interact with piston and liner walls, ${ }^{3}$ inkjet printing, ${ }^{4,5}$ droplet-based microfluidics, ${ }^{6,7}$ anti-icing, ${ }^{8}$ spray cooling,,${ }^{9,10}$ crude oil industry, ${ }^{11}$ etc. There are also many applications in chemical and petroleum processing, such as fluid catalytic cracking (FCC) where atomized droplets are brought into contact with high temperature fluidized catalyst particles. ${ }^{12}$ Depending on the conditions of impact, occurrence of different phenomena, such as spreading, bouncing or splashing is possible. ${ }^{13}$ The determining factors for the post-impingement behavior are droplet size, impact velocity and direction, liquid properties (e.g. density, viscosity, and viscoelasticity), solid surface properties (e.g. roughness and wettability), surface or interfacial tension, and substrate geometry. ${ }^{14,15}$ These effects are best described and assessed when appropriate dimensionless groups are used. Weber (We) and Reynolds $(R e)$ numbers are mostly employed in this regard. Weber number represents the ratio of kinetic energy to surface energy $\left(\frac{\rho V_{0}^{2} D}{\sigma}\right)$ and is a measure of the deformability of the droplet; and Re number is the ratio of inertia to viscosity effect $\left(\frac{\rho V_{0} D}{\mu}\right) \cdot{ }^{16}$

In terms of wettability, the solid substrate is either hydrophilic (wettable) or hydrophobic (nonwettable). Hydrophobic surfaces (HS) and superhydrophobic surfaces (SHS) possess some unique and practical properties such as surface cleaning, ${ }^{17}$ anti-fouling, anti-icing, dropwise condensation ${ }^{18}$ and drag reduction. ${ }^{19}$ A superhydrophobic surface (SHS) is standardly defined as 
a surface with an equilibrium contact angle (CA) greater than $150^{\circ}$ and very low $\left(<10^{\circ}\right)$ contact angle hysteresis $(\mathrm{CAH}){ }^{20}$ The $\mathrm{CAH}$, defined as the difference between the advancing and receding CA, is often used as a measure of drop mobility on a surface. Smaller values of CAH denote higher mobility. ${ }^{21}$ The receding CA value for a hydrophobic surface (HS) is generally assumed to be in the range of $90^{\circ}$ to $140^{\circ}$. On the other hand, lower values of the equilibrium CA are attributed to hydrophilic surfaces.

A vast number of studies have been published that use experimental and numerical approaches to study the impact of droplets on solid HSs and SHSs. ${ }^{14}$ Upon landing of a droplet on a solid surface, the inertia causes the droplet to spread and then it starts to retract due to surface tension effects. ${ }^{22}$ The retraction phase of impact, when performed on SHSs, can lead to partial or complete rebound. ${ }^{23}$ Impact velocity has also been found as a determining factor for the occurrence of rebound, such that the velocity of impact has to be low enough to allow the droplet to bounce off the surface ${ }^{24}$ without splashing. Impact and rebound characteristics on HS and SHSs - which is the subject of the present study - have been investigated in numerous published studies. Chen et al. ${ }^{25}$ experimentally investigated the outcomes of impact on an artificial SHS as well as lotus leaves and found the critical impact velocity for the rebound to occur. Antonini et al. ${ }^{26}$ conducted experimental tests to understand conditions of drop rebound from HSs and SHSs. The authors found that the receding CA on a surface has to be greater than $100^{\circ}$ for rebound to occur. Their results additionally showed that as the value of receding CA increases, rebound time decreases. Yeong et al. ${ }^{27}$ conducted an experiment to investigate the impact and rebound dynamics on a nanocomposite SHS at various surface angles relative to the horizon and different liquid droplet viscosities. Their results demonstrated that at low normal We numbers (We $\leq 30)$, no discernible difference exists between normal and oblique impacts in terms of maximum drop spread and 
rebound dynamics. A numerical investigation of the droplet impact dynamics on the SHS carried out by Bange and Bhardwaj ${ }^{13}$ revealed that the droplet bounces off the surface only if its total energy at the instant of maximum recoiling exceeds the initial surface and gravitational energy.

In terms of surface curvature, the solid substrates can be divided to flat (planar) and curved (nonplanar). The impact onto curved surfaces (spherical or cylindrical) has recently attracted the attention of the researchers since there are a number of applications such as particle coating (e.g., in pharmaceutical processes), as well as modification of surface roughness, ${ }^{28}$ drop impact onto wires, cables or antennas, and a better description of icing effect and spray coatings. Another situation where this phenomenon can be observed is, for example, in Multi-Effect Desalination (MED) evaporators, where sea water is sprayed on a tube bundle carrying hot steam in order to obtain distilled water inside the tubes. One of the earliest studies was performed experimentally by Levin and Hobbs ${ }^{29}$ in 1971 on water drop splashing behavior upon impacting onto a curved wall, whose radius of curvature is much larger than the drop. Later, an experimental study of droplets impacting on cylindrical wires was conducted by Hung and Yao. ${ }^{30}$ Based on their results, depending on the impact velocity and the relative size of the droplet and the wire, the impact outcome are disintegration and dripping. For instance, disintegration is observed primarily at moderate impacting velocities and for wires smaller than the incoming droplet; and dripping usually occurs when the wire is larger than the droplet size. Eral et al. ${ }^{31}$ used electrowetting to change the CA of a water drop deposited on a sphere with a comparable radius of curvature. The aim was to find the equilibrium morphology of the drop as a function of the CA and the drop volume. Mitra et al. $^{32}$ theoretically and experimentally investigated the process of droplets impacting on a highly thermally conductive spherical surface. Through their investigations, the authors varied different parameters such as droplet liquid, We number, and surface temperature. 
The authors observed that increased We numbers are accompanied with increased spread diameters and consequently, increased contact area, which in turn causes the heat transfer efficiency to improve. More recently, the dynamics of drop impact onto a sphere at moderate $R e$ and We numbers were investigated numerically by Zhu et al.. ${ }^{1}$ They quantitatively evaluated the effect of the aspect ratio of the sphere to the drop on the dynamics of drop spreading and retraction and presented a relation to approximate the thickness of the liquid film spreading on the sphere. Andrew et al. ${ }^{33}$ employed numerical simulations to investigate the contact time and bouncing of droplets on a semi-cylindrical ridge of varying size. The authors found that as the ridge radius decreases, corresponding to an increased curvature, the contact time is reduced. Furthermore, for ridges smaller than the drop, there is a pinch-off point which splits the drop into two smaller ones.

Our previous numerical study of droplets impacting onto spherical solid HSs and SHSs ${ }^{34}$ dealt with impact characteristics such as the contact time. It was found that a gap exists in literature concerning the bouncing behavior of droplets on curved surfaces and the models that can predict the impact outcomes. Moreover, the numerical studies in the literature are still mostly conducted 2-dimensionally with a focus on impacts in which the spherical surface radius is larger than the droplet radius.

Using 3D level set method simulations, the present study aims to examine the effects of a wide range of parameters on the outcome of the droplet impact, such as the size ratio of the solid surface to the droplet diameter, the liquid surface tension, the surface hydrophobicity, and the impact We number to obtain a more comprehensive understanding of their relative effects. Ultimately, to the authors' knowledge, no model or relationship exists in literature that predicts the spreading diameters of droplets impacting on curved surfaces. Therefore, in this paper, through analyzing the results of the simulations, it is attempted to propose a model which fulfills this purpose. 


\section{Mathematical Model and Governing Equations}

A single set of governing equations is solved to obtain the flow field, including continuity equation

as:

$$
\nabla \cdot \vec{u}=0
$$

and momentum equation as:

$$
\rho\left(\frac{\partial \vec{u}}{\partial t}+\vec{u} \cdot \nabla \vec{u}\right)=-\nabla p+\nabla\left(\mu\left(\nabla \vec{u}+(\nabla \vec{u})^{T}\right)\right)+\rho \vec{g}+\sigma \kappa \delta \vec{n}+\vec{F}
$$

where the symbols $\rho, \mu$ and $p$ denote the density, dynamic viscosity and pressure, respectively. The surface tension force which acts on the interface between the two phases is dealt with by the $(\sigma \kappa \delta \vec{n})$ term; where $\sigma$ is the surface tension coefficient, $\delta$ is the delta function concentrated at the interface between the two fluids, $\vec{n}$ is unit normal vector to the interface pointing into the droplets, and $\kappa$ is the local curvature of the fluid-fluid interface. ${ }^{4}$ Furthermore, the last term in equation (2) shows the effect of external forces, in this case, the gravitational force.

The local interfacial curvature $(\kappa)$ can be defined as: ${ }^{35}$

$$
\kappa=\nabla \cdot \vec{n}=\nabla \cdot \frac{\nabla \Phi}{|\nabla \Phi|}
$$

To track the fluid interface, the level set method (LSM) is employed. A conservative LSM is utilized in this study which has been proposed by Olsson and Kreiss. ${ }^{36}$ The conservative form helps to overcome the problems confronted when using conventional LSM, such as incorrect mass gain or loss in regions with severe interface deformation. The interface is represented by $\Phi=0.5$, where $\Phi$ is the level set function. The level set equation is defined as: 


$$
\frac{\partial \Phi}{\partial t}+\vec{u} \cdot \nabla \Phi=\gamma\left[\nabla \cdot\left(\varepsilon \nabla \Phi-\Phi(1-\Phi) \frac{\nabla \Phi}{|\nabla \Phi|}\right)\right]
$$

where $\epsilon$ and $\gamma$ denote the artificial thickness of the interface and the re-initialization parameter, respectively. The right-hand side of this equation consists of two terms. The first term is the divergence of $\epsilon \nabla \phi$ which acts as an artificial diffusivity to prevent discontinuities at the interface and the second term is a compressive flux. ${ }^{37}$ Furthermore, the level set function is utilized for smoothing the fluid properties across the interface. To illustrate, the density and the viscosity are calculated at each control volume by Equations 5 and 6, respectively:

$$
\begin{aligned}
& \rho=\rho_{1}+\left(\rho_{2}-\rho_{1}\right) \Phi \\
& \mu=\mu_{1}+\left(\mu_{2}-\mu_{1}\right) \Phi
\end{aligned}
$$

where the indices 1 and 2 represent the continuous and the dispersed phases, respectively.

The finite-element based solver COMSOL Multiphysics, is used here to solve Equations (1) - (6). The simulations are performed using static contact angle model. Droplet impacts on HSs and SHSs at low We can be predicted reasonably using static contact angle model; and cases with such characteristics are validated with experimental results in the following section.

\subsection{Validation}

In order to validate the numerical scheme used in this study, a few cases whose experimental data are available in literature have been considered. As the first case, a qualitative validation is provided by employing the results of an experimental study by Wang et al.. ${ }^{38}$ The time-lapsed images of a water droplet impacting on a SHS with an impact velocity of $0.56 \mathrm{~m} / \mathrm{s}$ and a CA of $163^{\circ}$ are compared in Figure 1, between the results of the current study and those presented experimentally by Wang et al.. ${ }^{38}$ 
(a)

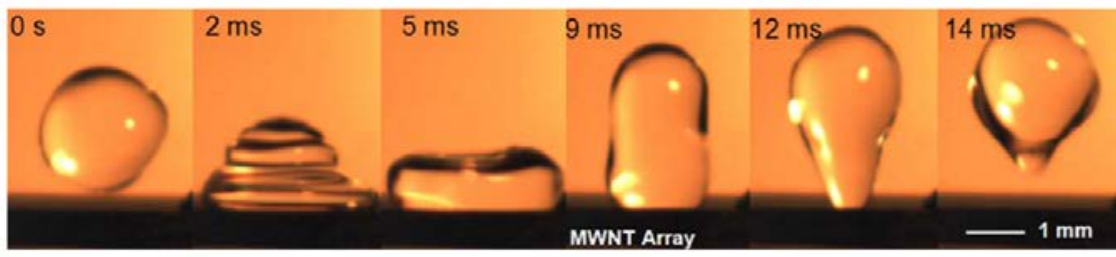

(b)

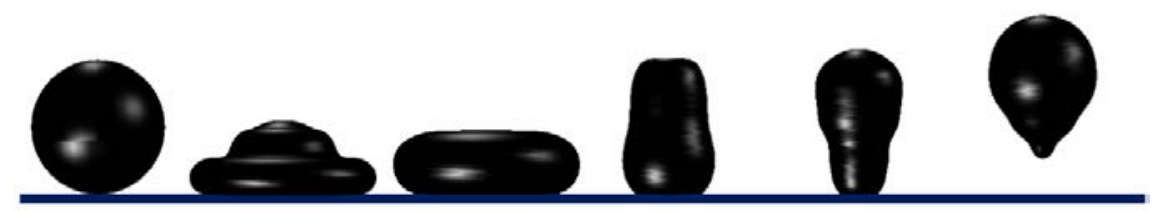

Figure 1. A water droplet impacting on a flat SHS as obtained by (a) Wang et al., ${ }^{38}$ and (b)

numerical simulations of the present study.

Additional validation is obtained using the experimental data of Kim et al.. ${ }^{39}$ A plot of the drop's normalized diameter versus time is presented in Figure 2, and a comparison is made between the experimental and the numerical data for two different impact velocities..

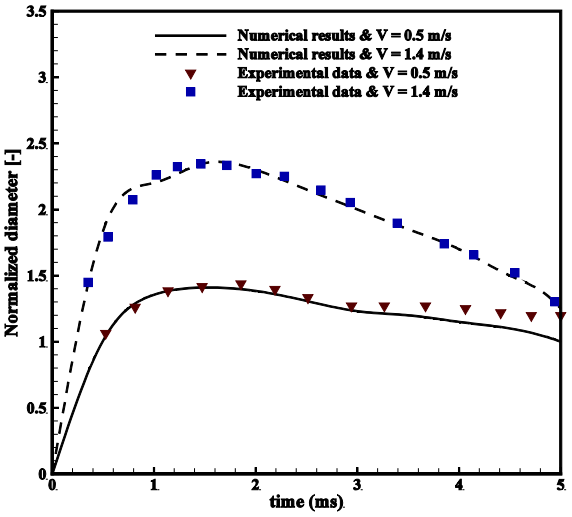

(a)

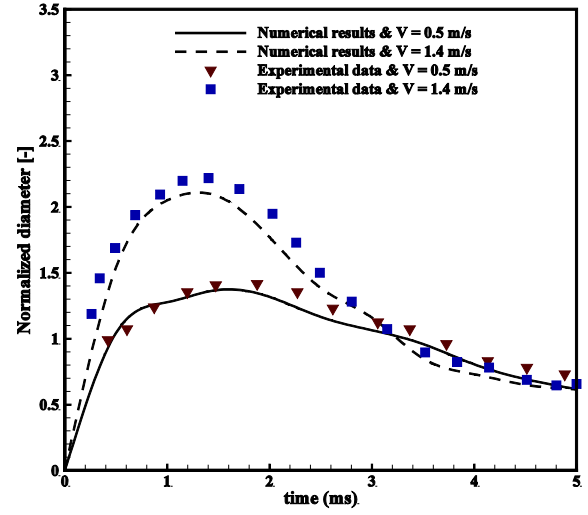

(b)

Figure 2. Normalized diameter of the droplet versus time for a CA of (a) $121^{\circ}$ and (b) $164^{\circ}$. 
The normalization is achieved through dividing the wetted diameter by the initial diameter of the droplet. As can be observed in these figures, acceptable agreement exists between the experimental results and those obtained using the numerical model, and this numerical model can predict the dynamics of drop impact accurately

\section{Model description}

All the cases considered in the present study consist of a liquid droplet impacting on a solid spherical substrate. An illustration of the pre-impact status of the system for a sample case studied, can be found in our previous work. ${ }^{34}$ The parameter $D^{*}$ is defined as the ratio of the solid sphere diameter to the droplet diameter. In all the cases considered for simulation, the droplet diameter will be kept constant and equal to $2.3 \mathrm{~mm}$, while $D^{*}$ will take values in the range 1 to 8 by varying the sphere diameter. The first part of the study is concerned with the impact of water droplets with properties given in Table 1 . In this table, $\mu_{0}$ is the zero-shear viscosity, $\gamma_{L V}$ represents surface tension between liquid and gas phases, and $\rho$ denotes the density.

Table 1. Physical properties of the water droplet ${ }^{40}$

\begin{tabular}{|c|c|c|c|}
\hline Material & $\mu_{0}(\mathrm{~Pa} \cdot \mathrm{s})$ & $\rho\left(\mathrm{kg} / \mathrm{m}^{3}\right)$ & \multirow{2}{*}{$\gamma_{L V}(\mathrm{~N} / \mathrm{m})$} \\
\cline { 1 - 3 } Water droplet & 0.001 & 998 & 0.072 \\
\hline Air & $1.814 \mathrm{e}-5$ & 1.204 & \\
\hline
\end{tabular}

The simulations are carried out in an axi-symmetrical domain. Initially, a perfectly circular droplet is assumed which is placed atop of the circular solid substrate such that the bottom of the droplet is in contact with the apex of the solid surface. A downward velocity (in the negative z-direction) is assigned to the droplet according to the considered We number in each simulation case. A gravitational force of $9.81 \mathrm{~m} / \mathrm{s}^{2}$ also acts along the negative $z$-direction. The systems is surrounded 
by air, with properties as listed in Table 1 . The considered cases include isothermal impacts of water droplets on HSs and SHSs with constant equilibrium CAs of $125^{\circ}$ and $163^{\circ}$, respectively. All simulations are carried out at a nominal temperature of $293.15 \mathrm{~K}$ and a pressure of $1 \mathrm{~atm}$, while the impact We number is varied from 5 to 30. In order to ensure the reliability and accuracy of the results, the computational domain must be sized in a manner that does not influence the convergence of the solution. The same holds true for the number of elements within each generated mesh. To this end, the simulation cases were tested to verify the independence of the results. The results of the tests for domain size and mesh elements are presented in Figures S1 and S2 respectively for a sample case. All other cases were treated similarly and the number of the elements for $D^{*}=1,2,4,6$ and 8 were chosen accordingly to equal 26655, 35215, 68670, 88433 and 119710 , respectively.

\section{Results and discussion}

A sample case is chosen to describe the dynamics of the impact process. The collision parameters include $W e=30, D^{*}=2$, and $C A=163^{\circ}$. The mentioned conditions lead to a complete drop rebound phenomenon, as shown in Figure 3a.
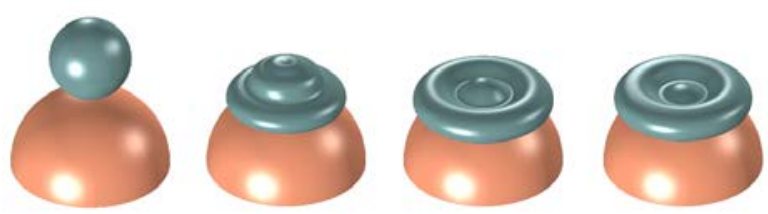

$t=0 \mathrm{~ms} \quad t=2 \mathrm{~ms} \quad t=4 \mathrm{~ms}$

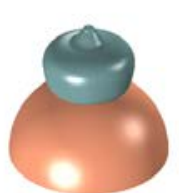

$$
t=6 m s
$$$$
t=8 m s
$$
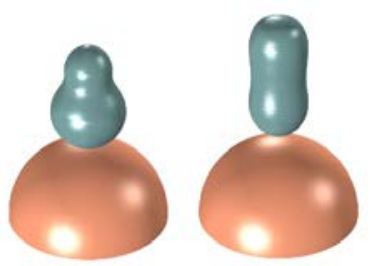

$$
t=10 \mathrm{~ms}
$$

$$
t=12 \mathrm{~ms}
$$

(a) 


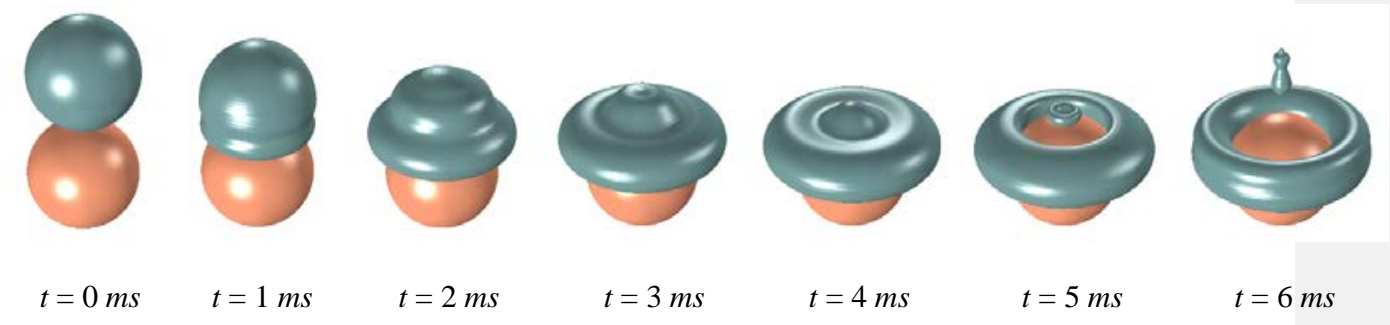

(b)

Figure 3. Shape deformation of the droplet upon impingement on a spherical SHSs with $W e=15$

$$
\text { and (a) } D^{*}=2 \text {; and (b) } D^{*}=1 \text {. }
$$

As can be observed in Figure 3a, the droplet starts spreading over the surface immediately after touching the solid surface. During this phase, despite the opposing effect of surface tension and viscosity of the fluid, the lower part of the liquid droplet in contact with the solid wall starts to stretch as the kinetic energy is converted to surface energy and viscous dissipation $(t=0 \mathrm{~ms}$ to $t=$ $4 \mathrm{~ms}$ ). This stage is accompanied with a larger mass being gathered at the outer edge of the liquid film. The deformation of the free surface is continued until the maximum spread diameter is reached when all the initial impact energy is converted to surface energy ( $t=4 \mathrm{~ms}$ ). At this instant, the energy stored in the droplet in the form of surface energy has to be consumed to provide the kinetic energy needed for beginning the retracting motion of the process ( $t=5 \mathrm{~ms}$ to $t=9 \mathrm{~ms}$ ). The retracting motion of the droplet towards its center, increases the pressure and prompts and upward movement in this region. The contact area keeps decreasing continuously and finally, a complete rebound is reached ( $t=10 \mathrm{~ms}$ ). As stated in our previous study, two factors contribute to the maximum spread diameter being larger for collision onto curved surfaces compared to the flat ones. These include the significant effect of centrifugal force and, to a lesser extent, the effect of 
gravity. For impacts on flat solid surfaces, unlike spherical or curved surfaces in general, the absence of centrifugal force is trivial and gravity does not have a component in radial direction.

Droplet impact onto spherical HSs will not always necessarily lead to complete rebound. In some cases, such as the one depicted in Figure 3b, disintegration of the droplet interface occurs. The collision parameters for the case depicted in Figure $3 \mathrm{~b}$ are $W e=15, D^{*}=1$, and $\mathrm{CA}=163^{\circ}$. As shown in this figure, the droplet starts to coat the solid sphere as soon as it is brought into contact with the surface and the same stages of spreading occur as described above. However, the spreading is followed by development of a neck area in the lamella as a result of the significant decrease in its thickness. At $t=5 \mathrm{~ms}$, the interface is disintegrated and a large liquid ring gets detached, leaving a very small fraction of the liquid atop the surface. It is found that this phenomenon is also observed for higher velocity impacts with the same size ratio $\left(D^{*}=1\right)$, but not for the impact with $W e=5$. Since this phenomenon is not observed for impacts on larger surfaces, its occurrence can be attributed to the augmented effect of curvature for smaller spherical surfaces. Similar observations have been reported by Ge and Fan ${ }^{41}$ and Yan-Peng and Huan-Ran. ${ }^{42}$

Cross-sectional images of the droplet and the solid surface with the velocity vectors during the lamella break-up is plotted in Figure 4a. This figure reveals that the velocity vectors acquire their highest magnitudes near the necked area and their deflection by the solid wall tends to separate the lower part of the droplet, where liquid mass is accumulated, from the apex of the droplet. By comparing Figures 4a and 4b for impacts with We numbers of 15 and 5, respectively, it can be observed that lamella thinning and break-up does not occur for the case with lower impact velocity. 


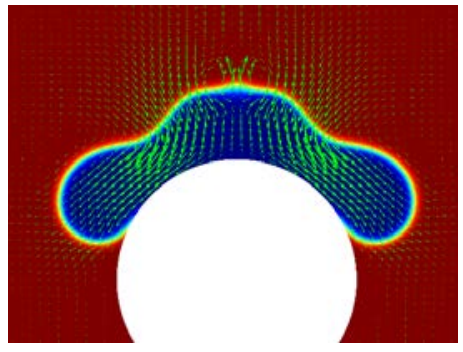

$t=3 \mathrm{~ms}$

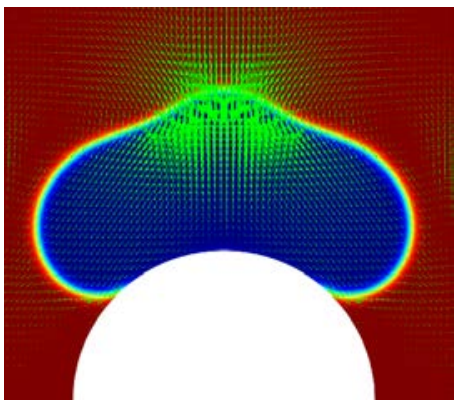

$t=4 \mathrm{~ms}$

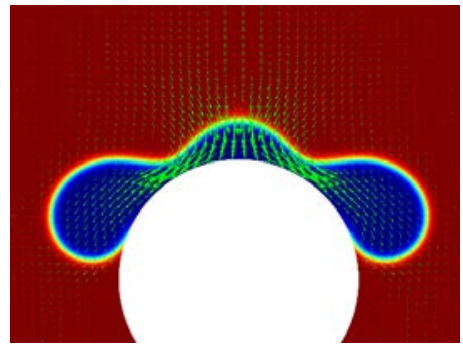

$t=4 \mathrm{~ms}$

(a)

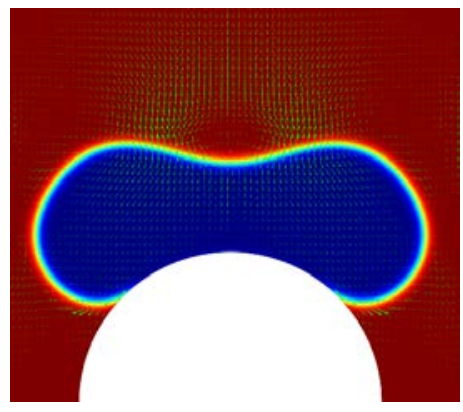

$t=5 \mathrm{~ms}$

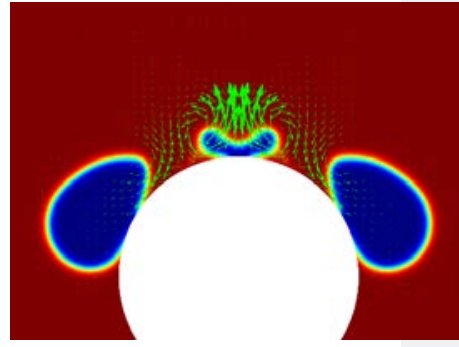

$t=5 \mathrm{~ms}$

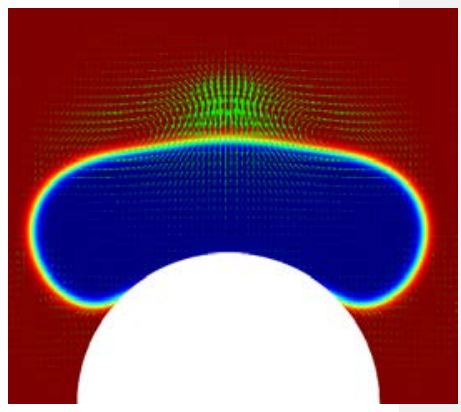

$t=6 \mathrm{~ms}$

(b)

Figure 4. Velocity vectors for the case of SHS with $D^{*}=1$ at (a) $W e=15$; and (b) $W e=5$ (the arrows lengths are proportional to the velocity).

In the following, the results of the simulations will be presented in separate parts, each part dealing with the effect a single parameter while all other parameters and conditions are kept constant and similar to each other.

\subsection{Effect of the surface hydrophobicity}

In this section, the effect of surface hydrophobicity on the water droplet impact outcome is assessed by plotting the normalized wetted diameter (dimensionless spread factor $\beta$ ) versus time and 
comparing it for two different CAs, while all the other impact conditions are the same. Figure 5 represents the influence of the CA for impacts of water droplets on surfaces with $D^{*}$ values of 2 and 6 at $W e=15$. A higher $\mathrm{CA}$ leads to a smaller maximum spreading diameter as well as a shorter duration of the spreading stage. This is in agreement with the experimental findings about drop impact on flat surfaces such as those reported by Kim et al.. ${ }^{39}$ For the case of impact on the surface with $D^{*}=6$, for instance, the maximum value of normalized diameter decreases by about $17 \%$ and the time for reaching the maximum spreading diameter is reduced from $4 \mathrm{~ms}$ to $3 \mathrm{~ms}$.

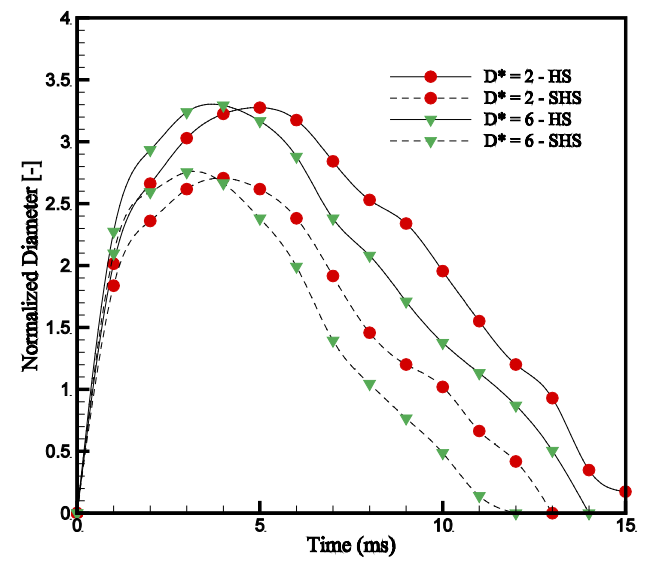

Figure 5. Time-varying spread factor of the droplet during impact onto surfaces with different

$$
\mathrm{CA} \text { at } W e=15 \text {. }
$$

\subsection{Effect of size ratio}

The ratio of the solid substrate diameter to the droplet diameter is another parameter which plays a key role in altering the spreading behavior of the droplet and its possible rebound. ${ }^{33}$ To investigate this, the impacts of water droplets on spherical SHSs with $D^{*}$ values of 2, 6 and 8 are compared in Figures $6 \mathrm{a}$ and $6 \mathrm{~b}$ at impact We numbers of 15 and 30, respectively. As can be 
observed from the figure, lower values of $D^{*}$ increase the spreading extent of the droplet and therefore, cause a higher proportion of the solid surface to be covered by the liquid. At lower impact velocities, the total impact time is slightly affected as well, where smaller spherical surfaces correspond to longer contact time between the droplet and the solid wall. In this case, as the size ration is varied from 2 to 8 , the impact time is reduced by $2 \mathrm{~ms}$. The authors also observed a similar trend for the case with $\mathrm{CA}=125^{\circ}$.

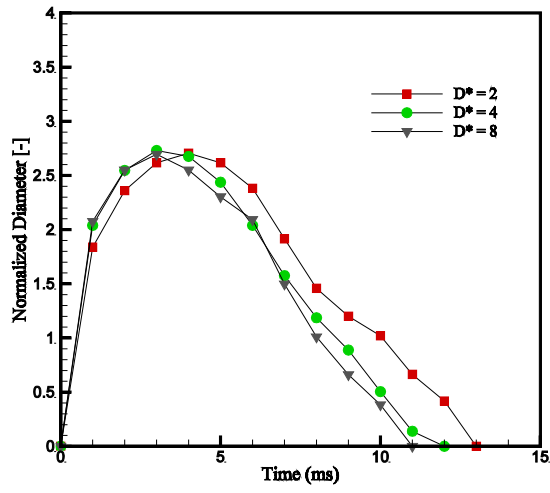

(a)

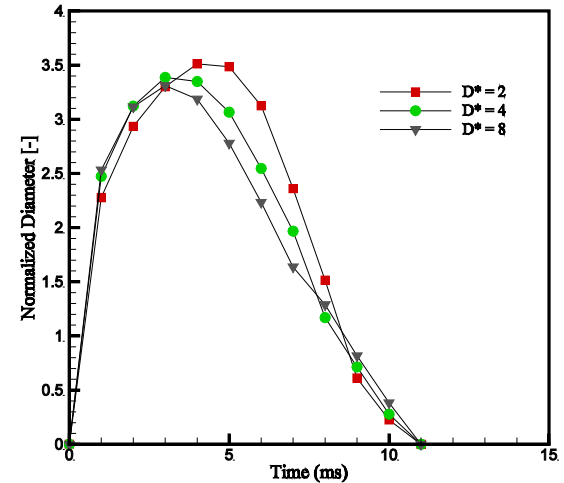

(b)

Figure 6. Time-varying spread factor of the droplet during impact onto SHSs with different surface-to-droplet size ratios when: (a) $W e=15$; and (b) $W e=30$.

\subsection{Effect of We number via varying impact velocity}

In order to investigate the outcomes of various impact velocities, different cases with low We numbers ranging from 5 to 30 are also included in the simulations. To illustrate the effect of impact velocity on the outcome of the droplet impact process, impacts with different velocities on spheres with $D^{*}=6$ have been considered and plotted for HSs and SHSs in Figure 7. As can be expected, the value of maximum spreading diameter increases considerably with the impact We number for 
the both surfaces with different CAs, which is due to the higher kinetic energy of the impacting droplet. Furthermore, the time for reaching the maximum value of spread factor becomes shorter as the impact velocity increases. It is also observed that generally, the duration of the recoiling phase of the impact is shorter at higher We numbers. This observation is understandable since higher impact velocities result in higher velocity during the receding phase; which has also been reported previously for impacts on flat surfaces by Rioboo et al.. ${ }^{43}$

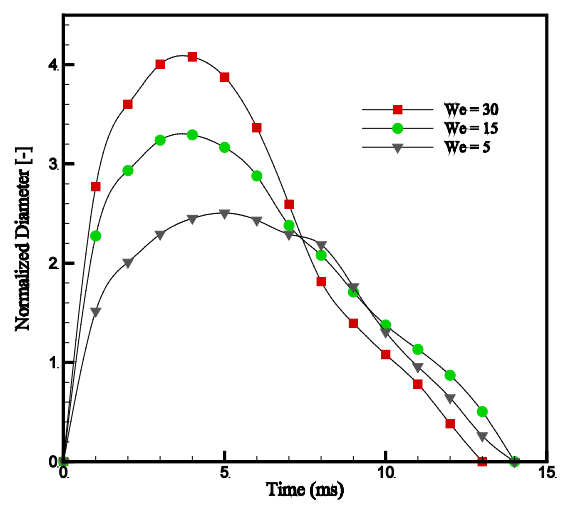

(a)

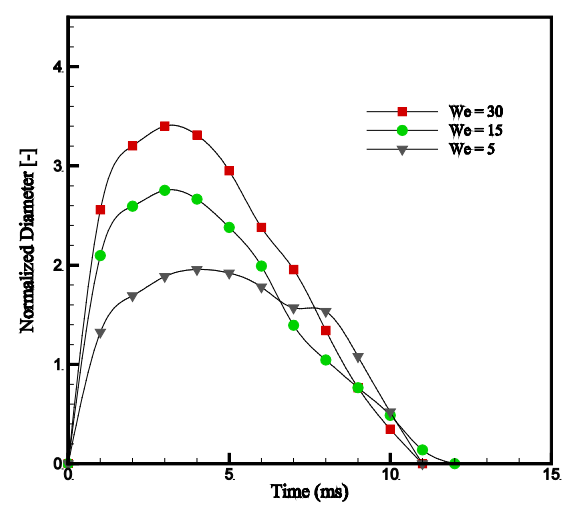

(b)

Figure 7. Time-varying spread factors of the droplets during impacts onto a solid sphere with $D^{*}$ $=6$ at different impact We numbers when: (a) $\mathrm{CA}=125^{\circ}$; and (b) $\mathrm{CA}=163^{\circ}$.

\subsection{Effect of We number via varying surface tension}

Surface tension is also a fundamental factor involved in determining whether the impact is concluded with a rebound. The effect of this parameter is studied by including two new synthetic fluids in the simulations. The only distinction between the properties of the new fluids and water is the values of the surface tension. While a fixed value of $0.072 \mathrm{~N} / \mathrm{m}$ was assumed in the simulations of water droplets impacting the solid surface, the new fluids are assumed to have $\gamma_{L V}$ 
values of 0.036 and $0.018 \mathrm{~N} / \mathrm{m}$, respectively. Figure 8 depicts the influence of surface tension by comparing impacts of droplets with different surface tension values on a solid spheres at impact $W e=5$. Decreasing the surface tension leads to more drastic shape deformations and therefore, higher values of maximum spread factors are accrued for both HSs and SHSs, as shown in Figure 8. It must be considered that obtaining high contact angles becomes a very difficult task as the surface tension is lowered.

Since the rebound phase is essentially initiated under the restoring action of the surface tension force, it is not unexpected that surface tension affects the recoiling phase more intensely than the spreading phase. Taking the impacts on the HS as an example, it can be observed in Figure 8b that the difference between the cases with the highest and the lowest values of surface tension in terms of the spreading phase duration is $7 \mathrm{~ms}$, while this time difference becomes $18 \mathrm{~ms}$ for the recoiling phase.

With lower values of the surface tension, there exist situations during the recoiling phase, when the normalized diameter attains a minimum value, but the droplet starts spreading again afterwards. This is due to the fact that with lower values of the surface tension, a relatively smaller amount of energy is able to be stored as surface energy and then converted back into kinetic energy during the recoiling phase of the impact. ${ }^{22}$ Therefore, at sufficiently low surface tensions, the surface tension energy released is not sufficient to support the rebound. This phenomenon can be noticed in Figure 8c for impacts on HSs with $D^{*}=4$ at $W e=5$, where the cases with $\gamma_{L V}$ values of 0.036 and $0.072 \mathrm{~N} / \mathrm{m}$ lead to a complete rebound, while the one with $\gamma_{L V}=0.018 \mathrm{~N} / \mathrm{m}$ attains a minimum value of normalized diameter at $t=30 \mathrm{~ms}$ and subsequently starts to spread again. 


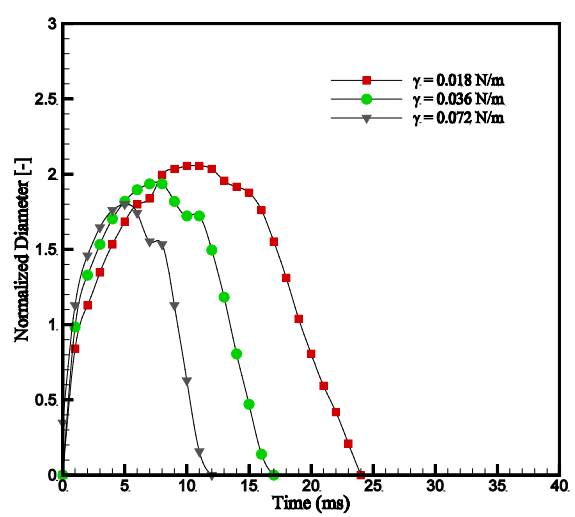

(a)

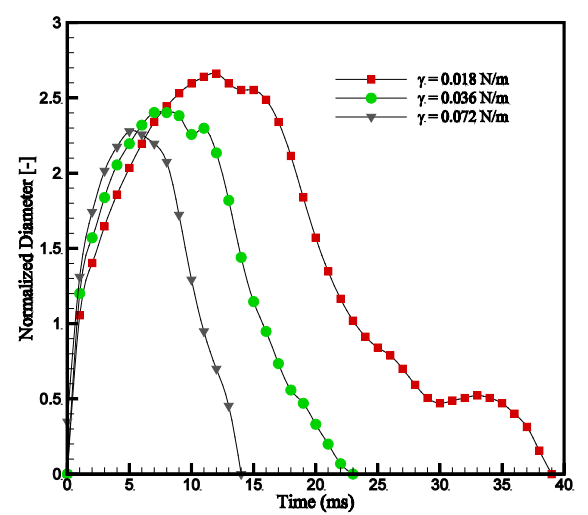

(b)

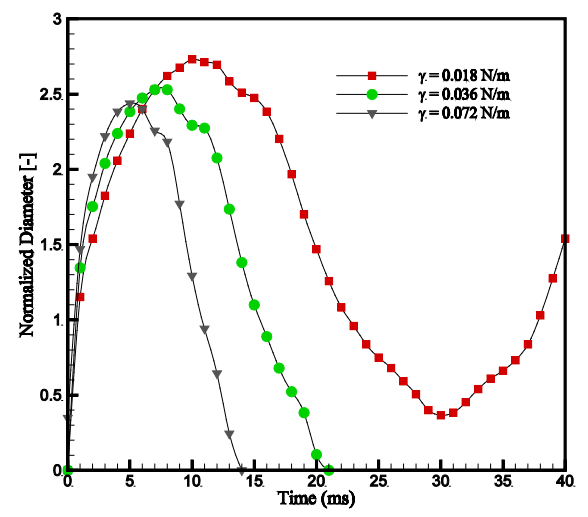

(c)

Figure 8. Time-varying spread factors of droplets during impacts onto solid spheres at impact We $=5$ when (a) $D^{*}=2$ and $C A=163^{\circ}$; and (b) $D^{*}=2$ and $C A=125^{\circ}$; and (c) $D^{*}=4$ and $C A=$ $125^{\circ}$

\subsection{A model to predict the maximum spreading diameter}

Maximum spreading diameter or radius is an important quantity that finds applications in several practical fields such as inkjet printing and forensic analysis. ${ }^{23}$ Numerous correlations have been developed hitherto by researchers to predict the maximum spreading diameter using important dimensionless parameters such as We and/or Re number, including those by Akao et al., ${ }^{44}$ Scheller 
and Bousfield, ${ }^{45}$ Clanet et al., ${ }^{46}$ and Roisman. ${ }^{47}$ For highly viscous droplets, Re number plays a more crucial role, while the inertial-dominated impacts are usually dealt with We number. The common aspect of the models currently available in literature is that they are developed based on the results of impacts onto planar surfaces. As discussed previously, the outcome of the impact onto curved surfaces is highly affected by additional forces including gravity and centrifugal force. For this reason, the values of maximum spreading diameter obtained from droplet impact onto curved surfaces will be different to a considerable extent from planar surfaces. The incapability of flat surface correlations in predicting the maximum spreading diameter of the droplets impacting on the spherical surfaces has been previously proved by the authors. ${ }^{34}$ In order to overcome this inadequacy, it is attempted here to propose a reliable and rather simple formula which can reasonably estimate the maximum spreading diameter of low-viscous (water in this case) droplets upon impacting on spherical HSs and SHSs.

To begin with, a parameter $\beta_{\max }$ is introduced to define the normalization of the maximum spreading diameter of the droplet by its initial diameter before coming into contact with the solid dimensionless spreading ratio as beta and the maximum spreading diameter as beta, $\max$ surface. Several cases are simulated, which include impacts of water droplets on spherical HSs and SHSs (CAs of $125^{\circ}$ and $163^{\circ}$, respectively) with $D^{*}$ values of $1,2,4,6$, and 8 for both hydrophobicity values. The simulations are performed at We numbers ranging from 5 to 30 . In addition, impact of a water droplet with its low viscosity on a HS or SHS suggests that the fraction of the kinetic energy which is dissipated by viscosity during the impact is minimal. Therefore, it can be argued that a correlation independent of Re number may be proposed for these conditions. Based on the discussed details, the values of $\beta$ for the above-mentioned cases are plotted versus We number in Figure 9a. As can be observed, the value of $\beta$ increases with We number and decreases with hydrophobicity. 
The values of $\beta$, as obtained from a number of pre-established models ${ }^{44-47}$ for impacts on flat surfaces, are presented in Figure 9b. The results presented in Figure 9a for impacts on spherical surfaces are also displayed in order to make a comparison possible. By comparing the results obtained from the models and the results obtained in the current study, it is obvious that the models developed for flat surfaces are not capable of properly predicting the maximum spreading diameter of the droplet upon impacting on the curved surfaces. Moreover, the mentioned correlations only depend on $R e$ and We numbers and therefore, cannot address the gap that exists between the results of impacts upon HSs and SHSs. Hence, a correlation needs to be proposed that takes into account the effects of the parameters We number and CA. Due to the low deviation of the results with changes in the size ratio of the solid surface to the droplet, the effect of this parameter will be ignored in developing the correlation. Through a comprehensive investigation, it is deduced that a power function can be fitted to the available data points. The derived formula, shown by dash-dot curves in Figure 10, is as follows:

$$
\beta=1.54 W e^{0.3}(3.52-\theta)^{0.25}
$$

where $\theta$ is the equilibrium CA in radians. This correlation is valid for low viscosity fluids and for a Weber number up to 30 . Inspecting the Figures $10 \mathrm{a}$ and $10 \mathrm{~b}$, it is observed that the proposed model predicts the spreading behavior at both values of hydrophobicity with a high level of reliability. Lastly, due to the fact that droplet impact on curved surfaces is highly sensitive to surface size and impact velocity which may lead to interface disintegration under different conditions, no asymptotic limit exists for the proposed correlation and caution has to be exercised in this regard when using the model. 


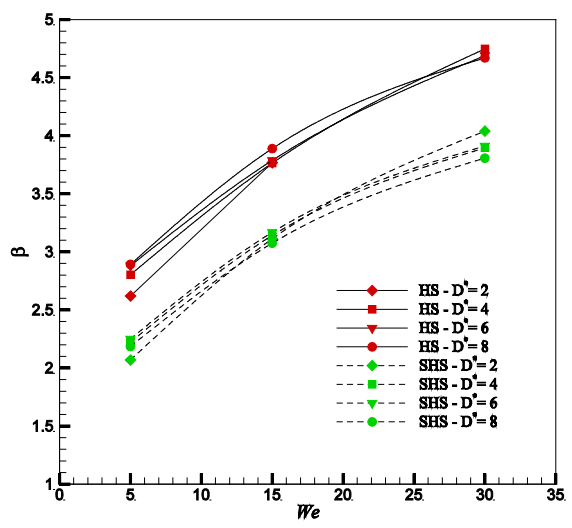

(a)

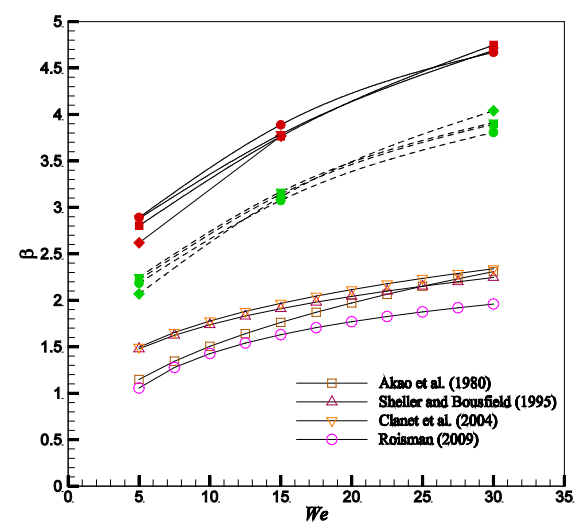

(b)

Figure 9. (a) Results of maximum spreading diameter versus We number obtained from the simulations; and (b) Comparison of these results to pre-established models for flat surfaces.

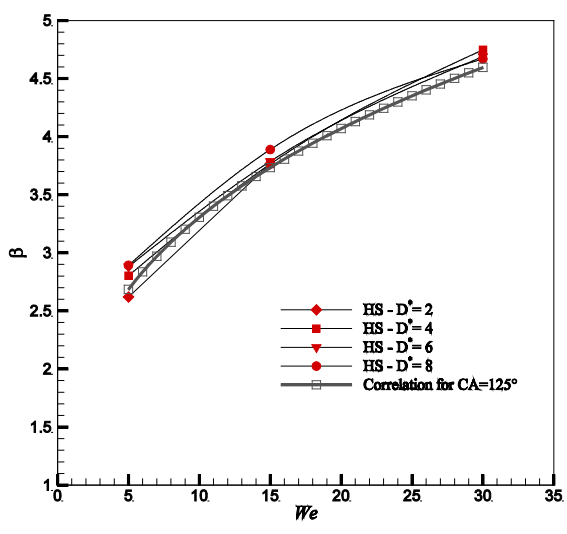

(a)

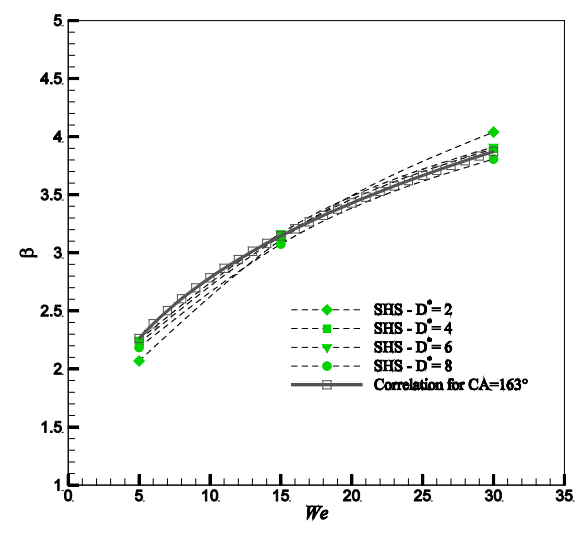

(b)

Figure 10. Comparison of maximum spreading diameter versus We number between the simulation results and the proposed correlation for CAs of (a) $125^{\circ}$; and (b) $163^{\circ}$.

\section{Conclusions}


A series of numerical simulations are performed in the present study to investigate the spreading and rebound behavior of a constant-size droplet impacting on spherical solid surfaces. HSs and SHSs with respective CAs of $125^{\circ}$ and $163^{\circ}$ are considered, whose diameters are equal to or larger than the droplet. Effect of various parameters on the impaction outcome are investigated. It is found that the maximum spreading diameter generally increases with impact velocity and decreases with increase in the diameter of the solid surface, the surface hydrophobicity, and the surface tension. The typical outcomes of the impact include complete rebound, disintegration of the interface, and arriving at a stable position on the surface after a series of fluctuations. Small surface diameter and high impact velocities are the factors that contribute to interface disintegration, while stable positioning occurs at lower values of surface tension. Finally, a predictive model is proposed - probably for the first time - that acceptably estimates the maximum spreading diameter of the low-viscous droplets rebounding from spherical HSs and SHSs

\section{References}

(1) Zhu, Y.; Liu, H. R.; Mu, K.; Gao, P.; Ding, H.; Lu, X. Y. Dynamics of Drop Impact onto a Solid Sphere: Spreading and Retraction. J. Fluid Mech. 2017, 824, R3.

(2) Massinon, M.; De Cock, N.; Forster, W. A.; Nairn, J. J.; McCue, S. W.; Zabkiewicz, J. A.; Lebeau, F. Spray Droplet Impaction Outcomes for Different Plant Species and Spray Formulations. Crop Prot. 2017, 99, 65-75.

(3) Malgarinos, I.; Nikolopoulos, N.; Marengo, M.; Antonini, C.; Gavaises, M. VOF Simulations of the Contact Angle Dynamics during the Drop Spreading: Standard Models and a New Wetting Force Model. Adv. Colloid Interface Sci. 2014, 212, 1-20.

(4) Bordbar, A.; Taassob, A.; Zarnaghsh, A.; Kamali, R. Slug Flow in Microchannels: 
Numerical Simulation and Applications. J. Ind. Eng. Chem. 2018.

(5) Bordbar, A.; Taassob, A.; Kamali, R. Diffusion and Convection Mixing of NonNewtonian Liquids in an Optimized Micromixer. Can. J. Chem. Eng. 2018.

(6) Dehghan Manshadi, M. K.; Khojasteh, D.; Mansoorifar, A.; Kamali, R. Efficiency Enhancement of ICEK Micromixer by a Rectangular Obstacle. In annual meeting for 3rd Annual International Conference on New Research Achievements in Chemistry \& Chemical Engineering; Tehran, Iran, 2016.

(7) Khojasteh, D.; Dehghan Manshadi, M. K.; Mousavi, S. M.; Kamali, R. Droplet Impact on Superhydrophobic Surface under the Influence of An Electric Field. In annual meeting for 3rd Annual International Conference on New Research Achievements in Chemistry \& Chemical Engineering; Tehran, Iran, 2016.

(8) Khojasteh, D.; Kazerooni, M.; Salarian, S.; Kamali, R. Droplet Impact on Superhydrophobic Surfaces: A Review of Recent Developments. J. Ind. Eng. Chem. 2016, 42, 1-14.

(9) Khojasteh, D.; Mousavi, S. M.; Kamali, R. CFD Analysis of Newtonian and NonNewtonian Droplets Impinging on Heated Hydrophilic and Hydrophobic Surfaces. Indian J. Phys. 2017, 91 (5), 513-520.

(10) Taassob, A.; Kamali, R.; Bordbar, A. Investigation of Rarefied Gas Flow through Bended Microchannels. Vacuum 2018, 151, 197-204.

(11) Kamali, R.; Khojasteh, D.; Mousavi, S. M. Newtonian and Non-Newtonian Droplet Impact onto a Heated Hydrophobic Solid Surface. In the annual meeting for 24th Annual International Conference on Mechanical Engineering-ISME; Yazd, Iran, 2016. 
(12) Mitra, S.; Nguyen, T. B. T.; Doroodchi, E.; Pareek, V.; Joshi, J. B.; Evans, G. M. On Wetting Characteristics of Droplet on a Spherical Particle in Film Boiling Regime. Chem. Eng. Sci. 2016, 149, 181-203.

(13) Bange, P. G.; Bhardwaj, R. Computational Study of Bouncing and Non-Bouncing Droplets Impacting on Superhydrophobic Surfaces. Theor. Comput. Fluid Dyn. 2016, 30 (3), 211-235.

(14) Pasandideh-Fard, M.; Bussmann, M.; Chandra, S. Simulating Droplet Impact on a Substrate of Arbitrary Shape. At. Sprays 2001, 11 (4), 397-414.

(15) Yarin, A. L. DROP IMPACT DYNAMICS: Splashing, Spreading, Receding, Bouncing.... Annu. Rev. Fluid Mech. 2006, 38 (1), 159-192.

(16) Tsai, P.; Pacheco, S.; Pirat, C.; Lefferts, L.; Lohse, D. Drop Impact upon Micro- and Nanostructured Superhydrophobic Surfaces. Langmuir 2009, 25 (20), 12293-12298.

(17) Abolghasemibizaki, M.; Robertson, C. J.; Fergusson, C. P.; McMasters, R. L.; Mohammadi, R. Rolling Viscous Drops on a Non-Wettable Surface Containing Both Micro- and Macro-Scale Roughness. Phys. Fluids 2018, 30 (2), 23105.

(18) Abolghasemibizaki, M.; Mohammadi, R. Droplet Impact on Superhydrophobic Surfaces Fully Decorated with Cylindrical Macrotextures. J. Colloid Interface Sci. 2018, 509, 422 431.

(19) Regulagadda, K.; Bakshi, S.; Das, S. K. Morphology of Drop Impact on a Superhydrophobic Surface with Macro-Structures. Phys. Fluids 2017, 29 (8).

(20) Ferrari, M.; Benedetti, A. Superhydrophobic Surfaces for Applications in Seawater. Adv. Colloid Interface Sci. 2015, 222, 291-304. 
(21) Antonini, C.; Amirfazli, A.; Marengo, M. Drop Impact and Wettability: From Hydrophilic to Superhydrophobic Surfaces. Phys. Fluids 2012, 24 (10).

(22) Moevius, L.; Liu, Y.; Wang, Z.; Yeomans, J. M. Pancake Bouncing: Simulations and Theory and Experimental Veri Fi Cation. 2014.

(23) Josserand, C.; Thoroddsen, S. T. Drop Impact on a Solid Surface. Annu. Rev. Fluid Mech. 2016, 48 (1), 365-391.

(24) Aboud, D. G. K.; Kietzig, A. M. Splashing Threshold of Oblique Droplet Impacts on Surfaces of Various Wettability. Langmuir 2015, 31 (36), 10100-10111.

(25) Chen, L.; Xiao, Z.; Chan, P. C. H.; Lee, Y. K.; Li, Z. A Comparative Study of Droplet Impact Dynamics on a Dual-Scaled Superhydrophobic Surface and Lotus Leaf. Appl. Surf. Sci. 2011, 257 (21), 8857-8863.

(26) Antonini, C.; Villa, F.; Bernagozzi, I.; Amirfazli, A.; Marengo, M. Drop Rebound after Impact: The Role of the RecedingContact Angle. Langmuir 2013, 29 (52), 16045-16050.

(27) Yeong, Y. H.; Burton, J.; Loth, E. Drop Impact and Rebound Dynamics on an Inclined Superhydrophobic Surface Drop Impact and Rebound Dynamics on an Inclined Superhydrophobic Surface. 2014, No. September.

(28) Banitabaei, S. A.; Amirfazli, A. Droplet Impact onto a Solid Sphere: Effect of Wettability and Impact Velocity. Phys. Fluids 2017, 29 (6).

(29) Levin, Z.; Hobbs, P. V. Splashing of Water Drops on Solid and Wetted Surfaces: Hydrodynamics and Charge Separation. Philos. Trans. R. Soc. London. Ser. A, Math. Phys. Sci. 1971, 269 (1200), 555 LP-585.

(30) Hung, L. S.; Yao, S. C. Experimental Investigation of the Impaction of Water Droplets on 
Cylindrical Objects. Int. J. Multiph. Flow 1999, 25 (8), 1545-1559.

(31) Eral, H. B.; Manukyan, G.; Oh, J. M. Wetting of a Drop on a Sphere. Langmuir 2011, 27 (9), 5340-5346.

(32) Mitra, S.; Sathe, M. J.; Doroodchi, E.; Utikar, R.; Shah, M. K.; Pareek, V.; Joshi, J. B.; Evans, G. M. Droplet Impact Dynamics on a Spherical Particle. Chem. Eng. Sci. 2013, 100, 105-119.

(33) Andrew, M.; Liu, Y.; Yeomans, J. M. Variation of the Contact Time of Droplets Bouncing on Cylindrical Ridges with Ridge Size. Langmuir 2017, 33 (30), 7583-7587.

(34) Khojasteh, D.; Bordbar, A.; Kamali, R.; Marengo, M. Curvature Effect on Droplet Impacting onto Hydrophobic and Superhydrophobic Spheres. Int. J. Comut. Fluid Dyn. 2017, 31 (6-8), 310-323.

(35) Taassob, A.; Manshadi, M. K. D.; Bordbar, A.; Kamali, R. Monodisperse Non-Newtonian Micro-Droplet Generation in a Co-Flow Device. J. Brazilian Soc. Mech. Sci. Eng. 2017, 39 (6), 2013-2021.

(36) Olsson, E.; Kreiss, G. A Conservative Level Set Method for Two Phase Flow. J. Comput. Phys. 2005, 210 (1), 225-246.

(37) Bashir, S.; Rees, J. M.; Zimmerman, W. B. Simulations of Microfluidic Droplet Formation Using the Two-Phase Level Set Method. Chem. Eng. Sci. 2011, 66 (20), 47334741.

(38) Wang, Z.; Lopez, C.; Hirsa, A.; Koratkar, N. Impact Dynamics and Rebound of Water Droplets on Superhydrophobic Carbon Nanotube Arrays. Appl. Phys. Lett. 2007, 91 (2).

(39) Kim, H.; Lee, C.; Kim, M. H.; Kim, J. Drop Impact Characteristics and Structure Effects 
of Hydrophobic Surfaces with Micro- And/or Nanoscaled Structures. Langmuir 2012, 28 (30), 11250-11257.

(40) Moon, J. H.; Kim, D. Y.; Lee, S. H. Spreading and Receding Characteristics of a NonNewtonian Droplet Impinging on a Heated Surface. Exp. Therm. Fluid Sci. 2014, 57, 94 101.

(41) Ge, Y.; Fan, L. S. Droplet - Particle Collision Mechanics with Film-Boiling Evaporation. J. Fluid Mech. 2007, 573, 311-337.

(42) Yan-Peng, L.; Huan-Ran, W. Three-Dimensional Direct Simulation of a Droplet Impacting onto a Solid Sphere with Low-Impact Energy. Can. J. Chem. Eng. 2011, 89 (1), 83-91.

(43) Rioboo, R.; Tropea, C.; Marengo, M. Outcomes From a Drop Impact on Solid Surfaces. At. Sprays 2001, 11 (2), 12.

(44) Akao, F. Deformation Behaviors of a Liquid Droplet Impinging onto Hot Metal Surface. Trans. Iron Steel Inst. Japan 1980, 20 (11), 737-743.

(45) B. L. Scheller; Bousfield, D. W. Newtonian Drop Impact with a Solid Surface. AIChE Journal, 41(6) 1995, 41 (6), 1357-1367.

(46) Clanet, C.; Béguin, C.; Richard, D.; Quéré, D. Maximal Deformation of an Impacting Drop. J. Fluid Mech. 2004, 517, 199-208.

(47) Roisman, I. V. Inertia Dominated Drop Collisions. II. An Analytical Solution of the Navier-Stokes Equations for a Spreading Viscous Film. Phys. Fluids 2009, 21 (5), 1-11. 
Table of content graphic:

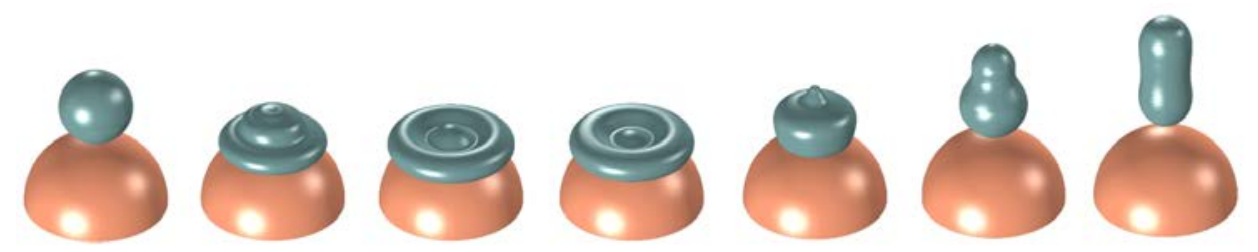

